“ (C) 2017 IEEE. Personal use of this material is permitted. Permission from IEEE must be obtained for all other uses, in any current or future media, including

reprinting/republishing this material for advertising or promotional purposes, creating new collective works, for resale or redistribution to servers or lists, or reuse of any copyrighted component of this work in other works." 


\section{Wideband Feeding Method for Full-Wave Dipole}

\author{
Can Ding, Bevan Jones, Haihan Sun, Pei-Yuan Qin, Y. Jay Guo \\ Global Big Data Technologies Centre (GBDTC) \\ University of Technology Sydney (UTS) \\ Sydney, NSW, Australia, 2007 \\ Can.ding@uts.edu.au
}

\author{
Luyang Ji \\ School of Electronics and Information \\ Northwestern Polytechnical University \\ Xi'An, Shannxi Province, China, 710071 \\ Luyangji@nwpu.edu.cn
}

\begin{abstract}
This paper introduces a wide-band feeding method for full-wave dipole antennas. A full-wave dipole is designed to cover the band from $698 \mathrm{MHz}$ to $960 \mathrm{MHz}$ for cellular base station applications. Its matching circuit consists of a laddertype filter design and a quasi-quarter-wavelength resistance transformer. The proposed matching circuit can provide balanced feeding as a balun and has a compact size. The matching circuit is designed and optimized using a circuit theory model and then physically realized using microstrip lines based on full-wave simulation. The simulated reflection coefficient $\left|S_{11}\right|$ is $<-\mathbf{- 1 5} \mathbf{d B}$ across the entire target band, exhibiting a bandwidth of $32 \%$.
\end{abstract}

\section{INTRODUCTION}

Half-wave dipole is one of the simplest and most widely used antennas in wireless communication and sensing. As well-known, a full-wave dipole has a higher gain and a narrower beamwidth than those of a half-wave dipole. Moreover, we notice that another significant advantage of full-wave dipoles is that they have a much broader bandwidth than halfwave dipoles of the same diameter in a similar configuration. For example, the $Q$ [1] of the dipole considered in this work as shown in Fig. 1 is 2.8 , whereas that of a half-wave dipole of the same diameter and height above the ground plane is 5.4. This indicates that, by simply increasing the dipole length from nearly half wavelength to nearly a wavelength, the dipole not only gets a narrower beamwidth but also a significantly widened bandwidth, on the premise that the full-wave dipole is well matched.

However, to the best of our knowledge, there are few published studies on full-wave dipoles. Offset-feeding [2,3] and dual/triple-feeding $[4,5]$ are the only available methods published in the literature. Offset-feeding is realized by moving the feed point away from the center of the dipole. Usually, the new feed point lies on one of the arms where the current reaches the peak. However, according to [3], the offset-feeding can deteriorate the radiation performance by introducing split lobes. Investigations in $[4,5]$ show that a full-wave dipole can be better fed by employing additional feed points. But both of these methods feature high fabrication complexity. Besides, none of these work is focused on the impedance bandwidth.

In this paper, a wideband center-fed matching circuit for anti-resonant full-wave dipoles is proposed. A straight-wire full-wave dipole is designed to demonstrate the proposed matching method. The matching circuit consists of a filter network and a resistance transforming network to tune the

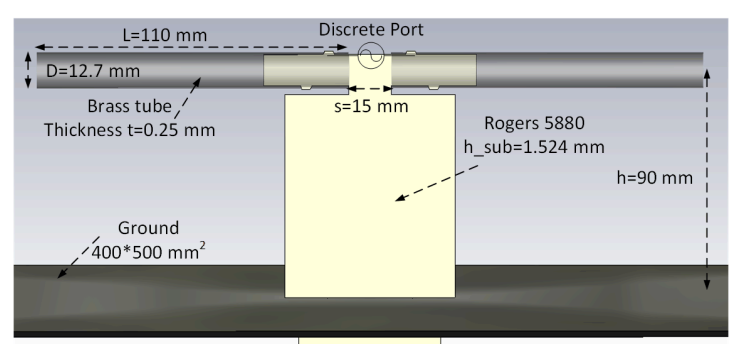

Fig. 1. Dipole configuration.

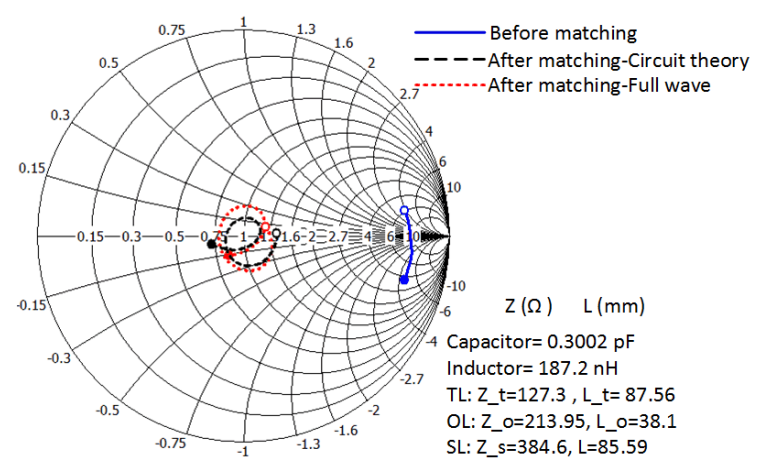

Fig. 2. Calculated input impedance of the dipole on Smith chart.

reactance and resistance, respectively. Although several components are employed to construct the matching circuit, the antenna remains compact due to the hybrid implementation method.

\section{Dipole And Matching Circuit Design}

Fig. 1 shows the full-wave dipole which is to be matched. The target band is from $698 \mathrm{MHz}$ to $960 \mathrm{MHz}$ (32\%), which is of interest for cellular base station applications. The calculated input impedance of the dipole before without matching circuit is plotted on a Z-smith chart in Fig. 2. The impedance of the dipole near its anti-resonant frequency can be approximated well by a parallel resonant R-L-C circuit where the resistance $\mathrm{R}$ represents radiation. The idea of impedance matching described in this work is to design a filter using serial and shunt resonant L-C circuits to tune the reactance and to use a quasiquarter-wavelength transmission line to tune the resistance as shown in Fig. 3. The filter has three stages. The first stage of 


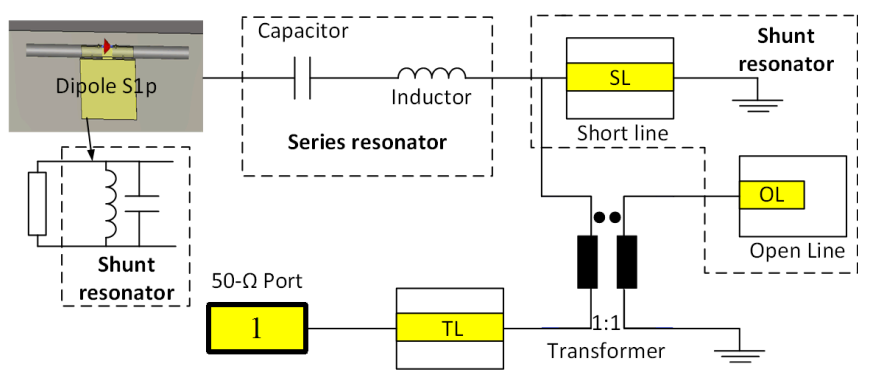

Fig. 3. Configuration of the matching circuit.

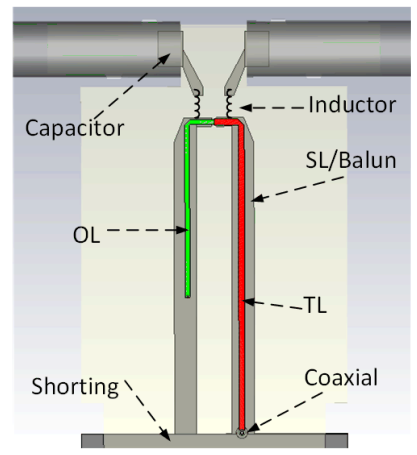

Fig. 4. Physical implementation of the matching network.

the filter in this arrangement is the shunt $\mathrm{L}-\mathrm{C}$ of the equivalent circuit of the dipole. Following this shunt resonator is a series L-C resonant circuit. The next shunt resonator of the filter is provided by a short line (SL) in parallel with an open line (OL). This three-stage ladder-type filter network is able to suppress the variation of the reactance in a very low level. To tune the resistance, a segment of transmission line (TL) nearly quarter-wavelength long is used. The quasi-quarterwavelength transmission line transformer is not sufficiently wideband for this application but the subsequent optimization allows compensating elements to improve the transformer's performance with the compensation of OL. After optimizing all the components in circuit model, the full-wave dipole is successfully matched across the target band with $\left|S_{11}\right|<-15$ dB, as shown in Fig. 2. Fig. 2 also gives the optimized values of the lumped components and the characteristic impedance $(\mathrm{Z})$ and the length $(\mathrm{L})$ of the transmission lines.

The physical implementation of the proposed matching circuit is shown in Fig. 4. The second-stage series resonator is constructed using lumped elements to minimize the space. More specific, the capacitors are realized by inserting copper strips into the tubes. The third-stage shunt resonator is implemented using distributed microstrip lines, which is attributed to two reasons. The first reason is that the resonator SL also functions as a balun and a balun made by lumped elements usually has narrow bandwidth. The second reason is that the quarterwavelength line transformer cannot accomplish an accurate resistance transformation of this magnitude across the required frequency band, without a compensation provide by the shunt resonator OL. The initial parameter values of the physical
TABLE I

COMPARISON BETWEEN THE FULL- AND HALF-WAVELENGTH DIPOLE

\begin{tabular}{|l|l|l|}
\hline Parameter & Full-wave dipole & Half-wave dipole \\
\hline Dipole length & $235 \mathrm{~mm}$ & $125 \mathrm{~mm}$ \\
\hline Directivity & $8.1-8.5 \mathrm{dBi}$ & $7.3-8.0 \mathrm{dBi}$ \\
\hline E-plane 3-dB Beamwidth & $60^{\circ}-63^{\circ}$ & $71^{\circ}-78^{\circ}$ \\
\hline H-plane 3-dB Beamwidth & $101^{\circ}-124^{\circ}$ & $103^{\circ}-122^{\circ}$ \\
\hline Antenna Q & 2.8 & 5.4 \\
\hline
\end{tabular}

matching components are derived from the optimization results in circuit simulation. Overall optimization and implementation tricks are required to transfer the circuit theory model to the 3D model. They are not included in this paper due to the page limit. Calculated $S_{11}$ of the dipole with matching circuit in CST Microwave 2016 full-wave simulation is also $<-15$ $\mathrm{dB}$ as shown in Fig. 2.

A comparison between the performance of the full-wave dipole in this work and a half-wave dipole in a similar configuration is presented in Table I. The results are obtained with an infinite ground plane placed $90 \mathrm{~mm}$ below the dipoles. As observed, the full-wave dipole has a higher directivity of about $0.5 \mathrm{dBi}$ on average. The major advantages of the fullwave dipole come from the intrinsic narrower 3-dB beamwidth in the E-plane and a smaller antenna Q (wider bandwidth).

\section{CONCLUSION}

In this work, a wideband matching method for full-wave dipoles is described. A straight wire full-wave dipole is well matched across the entire band from $698 \mathrm{MHz}$ to $960 \mathrm{MHz}$ with $\left|S_{11}\right|<-15 \mathrm{~dB}$. The matching circuit was optimized in circuit theory simulation and then implemented using microstrip technology and validated by full-wave simulation in CST Microwave Studio. The obtained full-wave dipole features broadband performance and stable radiation characteristics. It is demonstrated that full-wave dipoles, which are generally considered to be difficult to match, can be well matched using a compact matching circuit. With this matching issue solved, full-wave dipoles can be better candidates in some applications due to their narrower beamwidth and wider bandwidth compared to half-wave dipoles.

\section{REFERENCES}

[1] A. D. Yaghjian and S. R. Best, "Impedance, bandwidth, and Q of antennas," IEEE Transactions on Antennas and Propagation, vol. 53, No. 4, pp. 1298-1324, April 2005.

[2] S. H. Idris and C. M. Hadzer, "Analysis of the radiation resistance and gain of a full-wave dipole," IEEE Antennas and Propagation Magazine, vol. 36, No. 5, pp. 45-47, Oct. 1994.

[3] H. Hosono, K. Shibata, K. Saegusa and T. Takano, "The radiation characteristics and current distribution of a full wavelength dipole," 2014 IEEE Antennas and Propagation Society International Symposium (APSURSI), Memphis, TN, pp. 75-76, 2014.

[4] Y. Khraisat, K. Hmood and A. Anwar, "Analysis of the radiation resistance and gain of full-wave dipole antenna for different feeding design," Journal of Electromagnetic Analysis and Applications, Vol. 4 No. 6, 2012, pp. 235-242.

[5] Y. Khraisat, K. Hmood and A. Anwar, "The current distribution of symmetrical dual and triple feeding full-wave dipole antenna," Modern Applied Science, Vol. 5, No. 6, 2011, pp. 126-132. 